\title{
Epigenetic Regulation of Nostoc punctiforme ATCC 29133 in Response to Nitrogen Availability
}

\author{
Claire A. Shaw ${ }^{1}$, Charles G. Brooke ${ }^{1}$, Angel Avalos ${ }^{1}$, Matthew Blow², Nicole Shapiro², \\ Tanja Woyke ${ }^{2}$ and Matthias Hess ${ }^{1 \#}$ \\ ${ }^{1}$ University of California, Davis, CA, USA \\ ${ }^{2} U$.S. Department of Energy National Laboratory at the University of California Berkeley, Berkeley, CA, \\ USA \\ ${ }^{1 \# A d v i s o r}$
}

\section{ABSTRACT}

Atmospheric nitrogen is unavailable to plants and animals until it has been fixed by microorganisms like the facultative diazotroph Nostoc punctiforme. A better understanding of how genes related to the nitrogen metabolism of $N$. punctiforme are regulated is imperative to gaining a more complete understanding of the global nitrogen cycle. Prokaryotes, like eukaryotes, can regulate genetic transcription via DNA methylation. DNA methylation is an epigenetic regulatory process during which a methyl group is added to the DNA to promote or inhibit transcription of particular genes. Although its importance in prokaryotic gene regulation has been recognized, the mechanism, conditions and biological role of this process are still not understood in great detail. To investigate the potential role of DNA methylation for regulating nitrogen metabolism in N. punctiforme, we generated genome methylation profiles for the organism under contrasting nitrate availability. Here, we report the restriction modification system of Nostoc punctiforme ATCC 29133, along with its methylated genome sequence, under contrasting nitrate availability. The generated methylation profiles revealed increased methylation for enzymes associated with assimilatory nitrate reduction, suggesting that Nostoc punctiforme employs DNA methylation to regulate its nitrogen metabolism. To our knowledge, this is the first time these systems and mechanisms are reported at the genome scale for N. punctiforme.

\section{Introduction}

Nostoc punctiforme is a filamentous cyanobacterium that plays a key role in the global nitrogen cycle by fixing atmospheric nitrogen, in the process making nitrogen available for use by plants and animals otherwise unable to access this nitrogen. Despite the global importance of N. punctiforme as a nitrogen fixer, little is known about the regulation of its genes involved in nitrate assimilation. There are multiple known mechanisms, including DNA methylation, for the regulation of genes in both eukaryotes and prokaryotes. DNA methylation in eukaryotic cells was described for the first time in the 1940s (1), while methylation for gene expression regulation in bacteria was not described until the 1980s and it remains less well understood (2,3). Epigenetic gene regulation, like DNA methylation and restriction modification systems, allow prokaryotes to respond to environmental factors to adjust genetic transcripts, and ultimately, key proteins $(3,4)$. N. punctiforme utilizes an array of proteins in the process of nitrogen fixation, like nitrate and nitrite reductases and nitrate-nitrite transporters, all of which have the potential to undergo epigenetic regulation. Regulation of the genes encoding key proteins in $N$. punctiforme would allow for efficient control of the nitrogen fixation process, though little is known about the methylation patterns of this organism. To better understand the role of environmental nitrate in regulating the genome of $N$. punctiforme, the cyanobacteria was grown in the presence and absence of nitrate, followed up by a genome-wide analysis of methylation events. The 
nitrogen fixation methylation analysis was performed using genome and methylation data generated via PacBio SMRT sequencing. Results of this analysis indicated that methylation events were dependent on nitrate availability.

\section{Methods}

Nostoc punctiforme ATCC 29133 (PCC 73102) was obtained from the American Type Culture Collection (ATCC) and grown at $26^{\circ} \mathrm{C}$ in a corning vent cap tissue flask using BG-11 (ATCC 616 medium) with and without $\mathrm{NaNO}_{3}$ $(5,6)$. Cultures (in the presence and absence of $\mathrm{NaNO}_{3}$ ) were inoculated and grown in duplicates under a light/dark $(16 \mathrm{~h} / 8 \mathrm{~h})$ cycle. After $240 \mathrm{hrs} ., 10 \mathrm{~mL}$ of each culture were harvested during the light cycle. Cell pellets were resuspended in $1 \mathrm{~mL}$ fresh media for DNA extraction. DNA was extracted using the FastDNA ${ }^{\text {TM }}$ SPIN Kit for Soil (MP Biomedicals, Burlingame, CA) according to the manufacturer's protocol. DNA concentration was determined using a Qubit 2.0 fluorometer (Life Technologies, Grand Island, NY) according to the manufacturer's protocol.

DNA from $N$. punctiforme grown in the presence and absence of $\mathrm{NaNO}_{3}$ was sequenced at the Department of Energy's Joint Genome Institute using the Pacific Biosciences (PacBio) sequencing technology (7). A SMRTbell ${ }^{\mathrm{TM}}$ library was constructed and sequenced on the PacBio RS platform as described previously (8). Putative restriction systems in $N$. punctiforme's genome were identified using REBASE (9). DNA Modification detection and motif analysis were performed using PacBio SMRT analysis platform [protocol version=2.2.0 method=RS Modification and Motif Analysis] (10). Briefly, raw reads were filtered using SFilter, and filtered reads were aligned to the $N$. punctiforme reference genome (8) using BLASR-v1 (11). Modified sites were then identified through kinetic analysis of the aligned DNA sequence data (12), and subsequently grouped into motifs using MotifFinder-v1. These motifs represent the recognition sequences of methyltransferase genes active in the genome (13).

Methylation events were filtered by removing events with an event score lower than 30 (corresponding to a $p$-value of 0.001). Gene frame locations were identified in the BioCyc genome browser of N. punctiforme ATCC 29133. An additional $100 \mathrm{bp}$ were added to each side of the gene frames to catch any potential promoter related modifications. Gene specific methylation events were then located by filtering modification locations of the motif.gff files, generated by the SMRT pipeline, to the identified gene frame. The resulting data were then searched for motifs identified to be differentially represented between conditions. Methylation events were counted only if they occurred in both of the replicates within a treatment group.

\section{Results}

Methylation analysis revealed that genes coding for key enzymes of assimilatory nitrate reduction had a higher methylation status when nitrate was present (Figure 1); suggesting that Nostoc punctiforme might employ DNA methylation to regulate its nitrogen metabolism. Genes displaying methylation differences between the nitrate treatments (Figure 1) are all involved in key steps of nitrate metabolism: $n t c A$ encodes a promoter of the nirA operon, which produces a nitrite reductase (nirA) and a nitrate reductase (narB) (14). nrtD, an additional gene of the nirA operon, encodes an ATP-binding cassette transporter for nitrate and nitrite $(10,15)$, and also displayed differentiated methylation patterns in the presence and absence of nitrate. $n r t P$, which is not part of the nirA operon, encodes a nitrate-nitrite transporter similar to $n r t D$ (16). With this similarity in mind, it is not surprising that $n r t P$ follows a comparable methylation pattern to $n r t D$, despite $n r t P$ having a preference of nitrate over nitrite (16).

\section{Conclusion}

Since all genes belonging to the nirA operon (i.e., nirA, $n r t A B C D$ ), as well as $n r t P$, are integral to nitrogen fixation, it is unsurprising that differentiated methylation of these genes seems to be employed by $N$. punctiforme to fine tune expression and ultimately to control nitrogen metabolism based on nitrate availability. Results from this work provide 
a first, but still incomplete, glimpse into the regulatory mechanisms employed by $N$. punctiforme and further indicate how other nitrogen fixing microbes may respond to nutritional conditions. Microbes, such as N. punctiforme, play an integral part in globe nutrient cycles, making it imperative that we gain a better understanding of how these microbes regulate their own transcription for efficient contribution at a population level to these cycles. There is still much to understand about how prokaryotes regulate their metabolic and basic cellular functions via response to environmental factors, like nutrient availability. The methylation data presented in this work only explores one aspect of the methylome and thus still has much to provide in the way of new insights. The data is currently publicly available through Joint Genome Institute's Integrated Microbial Genomes and Microbiomes system, and should be further explored by the scientific community in order to provide all with a better understanding of how prokaryotes utilize DNA methylation to respond to environmental factors. We anticipate the data generated during this project and other data that are publicly available for download, along with further analysis through the Joint Genome Institute's Integrated Microbial Genomes and Microbiomes system, will provide a rich source for the scientific community to obtain additional insights into these mechanisms.

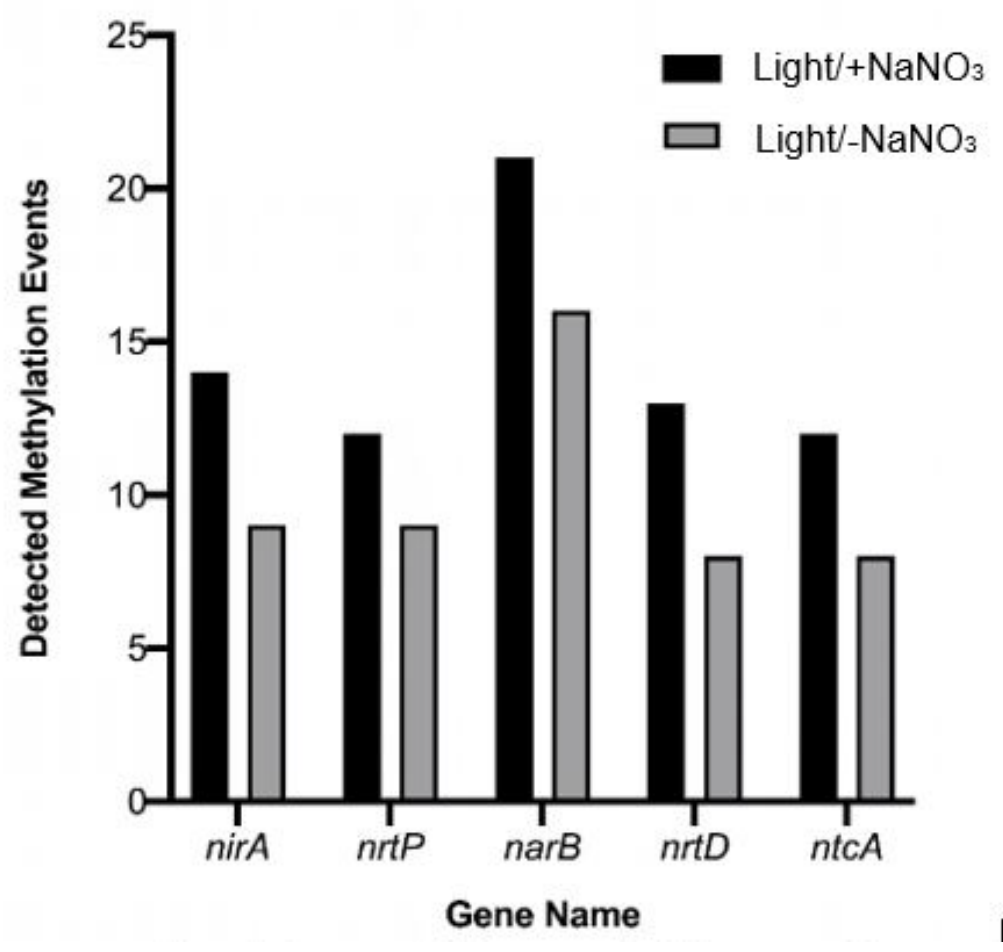

Figure 1. Effect of nitrate availability on methylation status of key genes of assimilatory nitrate reduction in Nostoc punctiforme ATCC 21933. Figure shows methylation status of genes encoding a nitrite reductase (nirA), nitrate/nitrite permease $(n r t P)$, nitrate reductase $(n a r B)$, a regulatory protein $(n t c A)$, as well as $n r t D$, which is part of the $n r t A B C D$ nitrate transporter operon (17). nirA, narB and $n r t D$ are part of the nirA operon (15).

\section{Data Availability}

The sequenced and annotated genome are publicly available through the Joint Genome Institute's Integrated Microbial Genomes and Microbiomes (IMG/M; https://img.jgi.doe.gov/cgi-bin/ mer/main.cgi) system (18) samples warehouse under the IMG Submission Identifier (ID) 62757. Methylation data can be found at https://genome.jgi.doe.gov/portal/pages/dynamicOrganismDownload.jsf?organism=TheNosnifixation. 


\section{Journal of Student Research}

\section{Acknowledgements}

The work conducted by the U.S. Department of Energy Joint Genome Institute, a DOE Office of Science User Facility, is supported by the Office of Science of the U.S. Department of Energy under Contract No. DE-AC02$05 \mathrm{CH} 11231$.

\section{References}

1. Moore L, Le T, \& Fan G. 2013. DNA Methylation and Its Basic Function. Neuropsychopharmacol 38: 23-38. https://doi.org/10.1038/npp.2012.112

2. Sternberg N. 1985. Evidence that adenine methylation influences DNA-protein interactions in Escherichia coli. Journal of Bacteriology. 164(1):490-493. DOI: 10.1128/jb.164.1.490-493.1985. PMID: 2995323; PMCID: PMC214274.

3. Barras F, Marinus MG. 1989. The great GATC: DNA methylation in E. coli. Trends Genet. 5(5):139-43. doi: 10.1016/0168-9525(89)90054-1. PMID: 2667217.

4. Casadesús J, Low D. 2006. Epigenetic gene regulation in the bacterial world. Microbiol Mol Biol Rev 70:830-856. doi: 10.1128/MMBR.00016-06.

5. Sanmartín P, Aira N, Devesa-Rey R, Silva B, Prieto B. 2010. Relationship between color and pigment production in two stone biofilm-forming cyanobacteria (Nostoc sp. PCC 9104 and Nostoc sp. PCC 9025). Biofouling. 26(5):499509. doi: 10.1080/08927011003774221. PMID: 20425659.

6. Rippka R, Deruelles J, Waterbury JB, Herdman M, Stanier RY. 1979. Generic Assignments, Strain Histories and Properties of Pure Cultures of Cyanobacteria. J Gen Microbiology. 111(1):1-61. doi: 10.1099/00221287-111-1-1

7. Eid J, Fehr A, Gray J, Luong K, Lyle J, Otto G, Peluso P, Rank D, Baybayan P, Bettman B. 2009. Real-time DNA sequencing from single polymerase molecules. Science 323:133-138. doi: 10.1126/science.1162986.

8. Moraes LE, Blow MJ, Hawley ER, Piao H, Kuo R, Chiniquy J, Shapiro N, Woyke T, Fadel JG, Hess M. 2017. Resequencing and annotation of the Nostoc punctiforme ATTC 29133 genome: facilitating biofuel and high-value chemical production. AMB Express 7:42. doi: 10.1186/s13568-017-0338-9.

9. Roberts RJ, Vincze T, Posfai J, Macelis, D. 2003. REBASE: restriction enzymes and methyltransferases. Nucleic Acids Res 31:418-420. doi: 10.1093/nar/gkg069.

10. Meeks JC, Elhai J, Thiel T, Potts M, Larimer F, Lamerdin J, Predki P, Atlas R. 2001. An overview of the genome of Nostoc punctiforme, a multicellular, symbiotic cyanobacterium. Photosynth Res 70:85-106. doi: 10.1023/A:1013840025518.

11. Chaisson MJ, Tesler G. 2012. Mapping single molecule sequencing reads using basic local alignment with successive refinement (BLASR): application and theory. BMC Bioinformatics 13:238. doi: 10.1186/1471-2105-13238. 


\section{Journal of Student Research}

12. Flusberg BA, Webster DR, Lee JH, Travers KJ, Olivares EC, Clark TA, Korlach J, Turner SW. 2010. Direct detection of DNA methylation during single-molecule, real-time sequencing. Nat Methods 7:461. doi: $10.1038 /$ nmeth.

13. Clark TA, Murray IA, Morgan RD, Kislyuk AO, Spittle KE, Boitano M, Fomenkov A, Roberts RJ, Korlach J. 2011. Characterization of DNA methyltransferase specificities using single-molecule, real-time DNA sequencing. Nucleic Acids Res 40:e29-e29. doi: 10.1093/nar/gkr1146.

14. Frías JE, Flores E. Induction of the Nitrate Assimilation nirA Operon and Protein-Protein Interactions in the Maturation of Nitrate and Nitrite Reductases in the Cyanobacterium Anabaena sp. Strain PCC 7120. J Bacteriol. 2015 Jul;197(14):2442-52. doi: 10.1128/JB.00198-15. Epub 2015 May 11. PMID: 25962912; PMCID: PMC4524197.

15. Frías J E, \& Flores E. 2010. Negative regulation of expression of the nitrate assimilation nirA operon in the heterocyst-forming cyanobacterium Anabaena sp. strain PCC 7120. Journal of bacteriology, 192(11): 2769-2778. doi: 10.1128/JB.01668-09

16. Aichi M, Yoshihara S, Yamashita M, Maeda S, Nagai K, Omata T. 2006. Characterization of the nitrate-nitrite transporter of the major facilitator superfamily (the nrtP gene product) from the cyanobacterium Nostoc punctiforme strain ATCC 29133. Biosci Biotechnol Biochem. 70(11):2682-9. doi: 10.1271/bbb.60286. Epub 2006 Nov 7. PMID: 17090931 .

17. Omata T. Structure, function and regulation of the nitrate transport system of the cyanobacterium Synechococcus sp. PCC7942. 1995. Plant Cell Physiol. 36(2):207-13. doi: 10.1093/oxfordjournals.pcp.a078751. PMID: 7767600.

18. Markowitz VM, Chen IM, Palaniappan K, Chu K, Szeto E, Grechkin Y, Ratner A, Jacob B, Huang J, Williams P, Huntemann M, Anderson I, Mavromatis K, Ivanova NN, Kyrpides NC. 2012. IMG: the Integrated Microbial Genomes database and comparative analysis system. Nucleic Acids Res 40 (Database issue):D115-D122. doi: $10.1093 / \mathrm{nar} / \mathrm{gkr} 1044$. 